\title{
A Dynamic Approach for Combining Abstract Argumentation Semantics
}

\author{
Jérémie Dauphin*, Marcos Cramer, and Leendert van der Torre*
}

\begin{abstract}
Abstract argumentation semantics provide a direct relation from an argumentation framework to corresponding sets of acceptable arguments, or equivalently to labeling functions. Instead, we study step-wise update relations on argumentation frameworks whose fixpoints represent the labeling functions on the arguments. We make use of this dynamic approach in order to study novel ways of combining abstract argumentation semantics. In particular, we introduce the notion of a merge of two argumentation semantics, which is defined in such a way that the merge of the preferred and the grounded semantics is the complete semantics. Finally we consider how to define new semantics using the merge operator, in particular how meaningfully combine features of naive-based and complete-based semantics.
\end{abstract}

\section{Introduction}

Following the methodology in non-monotonic logic, logic programming and belief revision, formal argumentation theory defines a diversity of semantics. This diversity has the advantage that a user can select the semantics best fitting her application, but it leads also to various practical challenges. First of all, how to choose among the considerable number of semantics existing in the argumentation literature for a particular application? The behaviour of semantics on examples can already be

Jérémie Dauphin

University of Luxembourg, Luxembourg; e-mail: jeremie.dauphin@uni.lu

Marcos Cramer

TU Dresden, Germany; e-mail: marcos.cramer@gmail.com

Leendert van der Torre

University of Luxembourg, Luxembourg; e-mail: leon.vandertorre@uni.lu

* The work of Jérémie Dauphin and Leendert van der Torre was supported by the H2020 Marie Skłodowska-Curie grant number 690974 for the project MIREL.

This is a preprint version from the authors. The published version can be found at: https://doi.org/10.1007/978-981-13-7791-4_2 
insightful, and Baroni and Giacomin [4] address the need for more systematic comparison of semantics based on a set of principles. However, what to do when no currently considered semantics is perfect? May there be a better semantics that has not been discovered yet? How to guide the search for new and hopefully better argumentation semantics? In this paper, we propose a new approach: the combination of abstract argumentation semantics. We focus on the following three research questions:

1. How to combine two abstract semantics to yield a third semantics?

2. In particular, how to obtain the complete semantics by combining the preferred and grounded semantics?

3. Can we meaningfully combine features of naive-based and complete-based semantics?

Concerning our first research question, there are various ways in which abstract argumentation semantics can be combined. For example, in multi-sorted argumentation $[13,1,12]$, one part of the framework can be evaluated according to for example grounded semantics, whereas another part of the framework is evaluated according to the preferred semantics. Another approach manipulates directly the sets of extensions. For example, the grounded and preferred can be combined by simply returning both the grounded and preferred extensions. Both of these approaches have drawbacks. For multi-sorted argumentation, we need to specify explicitly which semantics must be applied to which part of the framework. For the direct combination method, the approach seems too coarse-grained and the number of ways to combine semantics seems relatively limited.

We therefore introduce a dynamic approach in this paper, which is based on the labeling approach to argumentation semantics, in which the three labels in, out and undec are used. In our dynamic approach, we define step-wise versions of standard semantics based on epistemic labelings, which associate with each argument a nonempty set of labels from $\{$ in, out, undec $\}$. Intuitively, the set represents uncertainty about the label. We start with labeling each argument of the framework with the set $\{$ in, out, undec $\}$. This represents that we do not know the labeling yet. Then in each step we refine the labels by removing some of the labels. Finally we end up with a single label for each argument, and thus with a standard labeling. To represent the possibility of multiple extensions, the steps are not deterministic. The steps are represented by an abstract update relation, which mathematically is simply a binary relation among epistemic labelings. Note that there are many distinct update relations representing the same standard semantics, and it is this additional expressive power that we will use in our first approach to combining abstract argumentation semantics.

Concerning our second research question, it is well known that the grounded semantics outputs the smallest complete extension, and that the preferred semantics outputs maximal complete extensions [10]. This suggests that there is potential to recover all complete extensions using a mixture of the grounded and preferred semantics. Note that there may be complete extensions that are neither minimal nor maximal, and that it is therefore non-trivial to recover all the complete extensions 
using the grounded and the preferred semantics, without loosing any complete extensions. Though the derivation of the complete semantics from the grounded and preferred semantics does not serve any practical purpose, it serves to show that our dynamic semantic framework has sufficient expressive power to combine abstract semantics. We therefore pursue this second question to showcase our combination operation.

Note that we do not claim it to be possible to retrieve the full set of complete extensions from preferred and grounded extensions alone, as they do not provide sufficient information, even when represented as labelings. Indeed, some argumentation frameworks have the same preferred and the same grounded labelings, yet differ in their complete labelings. We present two such frameworks in Section 2. Hence, the approaches we propose still take the structure of the framework into account when combining the different semantics.

Concerning the third research question, note that recently naive-based semantics like stage semantics [15] and CF2 semantics [5] have received some attention, for example in the work of Gaggl and Dvořák [11], who define a new semantics (stage2) that combines features of stage and CF2 semantics, and in the work of Cramer and Guillaume [8], who performed an empirical study that showed that these naivebased semantics are better predictors of human argument acceptance than completebased semantics like the grounded and preferred semantics.

For argumentation frameworks without odd cycles, the stage semantics fully agrees with the preferred semantics. One difference between the preferred semantics and the stage semantics is that the stage semantics generally provides a way to select accepted arguments even when odd cycles are around, whereas the preferred semantics tends to mark as undecided all arguments that are in an odd cycle or attacked by an odd cycle. One difference between the preferred semantics and the complete semantics is that the complete semantics allows one to locally not make choices for some unattacked even cycles while making choices for other unattacked even cycles, whereas in the preferred semantics one has to make choices for all unattacked even cycles. This motivates the following research question: Is there a sensible semantics that allows one to locally make choices for some unattacked odd or even cycles while not making choices for other unattacked odd or even cycles?

The layout of this paper is as follows. After providing some preliminaries about argumentation semantics in Section 2, we introduces our dynamic approach based on epistemic labelings and update relations in Section 3. Section 4 addresses the second research question by showing how grounded and preferred semantics can be combined to obtain the complete semantics using an algorithmic approach to updates. As this approach is dependent on the choice of algorithm on which the update relation is based, we proceed in Section 5 to defining the merge of two argumentation semantics, a modification of our first approach that is applicable to any pair of semantics independently of any algorithmic considerations. In Subsection 5.1, we motivate the definition of the merge operator by considering how to use it to get the complete semantics from the grounded and preferred semantics without adding any algorithmic information. In Subsection 5.2, we show how the merge operator can be used to give rise to novel argumentation semantics, and, in particular, how it 
can be used to meaningfully combine features of naive-based and complete-based semantics. We conclude with an overview of further work in Section 6.

\section{Preliminaries}

An argumentation framework (AF) is a directed graph $\langle A, R\rangle$, where $A$ is called the set of arguments, and $R$ is called the attack relation. In this work, we do not consider enriched AFs such as bipolar AFs and weighted AFs. Standard argumentation semantics come in two variants. Extension-based semantics associates with each AF a set of extensions (sets of the arguments). Labelling-based semantics attribute to each argument the label in, out or undecided. The two approaches are inter-definable, in the sense that an argument is labeled in when it is in the extension, it is labeled out when it is not in the extension and there is an argument in the extension attacking it, and it is undecided otherwise. Our dynamic approach uses an epistemic labelling, which associates with each argument a nonempty set of labels. Intuitively, the set represents uncertainty about the label.

We assume familiarity with 3-labeling semantics of argumentation frameworks as defined in [3]. Note that we will make use of the multi-labeling approach, where a set of labels is assigned to each argument. This set represents the possible labels for a given argument. The standard approach corresponds to the case where arguments are given singleton sets as labels.

We define $\mathbb{L}=\{$ in, out, undec $\}$ to be the set of possible labels.

Definition 1. Let $F=\langle A, R\rangle$ be an AF. We say that any function $L$ from $A$ to $\mathbb{L}$ is a 3-labeling of $F$.

The 3-labeling approach makes use of the notions of legal labels.

Definition 2. Let $F=\langle A, R\rangle$ be an AF, $a \in A$ an argument and $L$ a 3-labeling of $F$. We say that $a$ is:

- legally in with respect to $L$ iff $L(a)=i n$ and for all $b \in A$ such that $(b, a) \in R$, $L(b)=$ out;

- legally out with respect to $L$ iff $L(a)=$ out and for some $b \in A$ such that $(b, a) \in R$, $L(b)=i n$;

- legally undecided with respect to $L$ iff $L(a)=$ undec and for all $b \in A$ such that $(b, a) \in R, L(b) \neq i n$ and for at least one such $b, L(b)=$ undec.

If all arguments in $A$ are legally labeled with respect to $L$, then we say that $L$ is a complete labeling of $F$. A complete labeling with a minimal set of in-labeled arguments is called a grounded labeling. A complete labeling with a maximal set of in-labeled arguments is called a preferred labeling. A complete labeling without undec-labeled arguments is called a stable labeling. A complete labeling with a minimal set of undec-labeled arguments is called a semi-stable labeling. 
An argumentation semantics is a function that maps an argumentation framework to a set of labelings. The above defined notions give rise to the complete, preferred and grounded argumentation semantics. We call an argumentation semantics $\sigma$ complete-based if all $\sigma$-labelings are complete labelings.

We will also refer to the stage semantics defined in its extension-based form by Verheij [15]. We adapt it to the labeling-based form by assigning the label out to all arguments that are not in the stage extension in Verheij's definition, even those which are not attacked by in arguments. This labeling-based form of the stage semantics can be defined as follows:

Definition 3. Let $F=\langle A, R\rangle$ be an AF and $L$ a 3-labeling of $F$. Define $L_{i n}$ to be the set $\{a \in A \mid L(a)=i n\}$. Define $L_{i n}^{+}$to be the set $\left\{a \in A \mid \exists b \in L_{i n} .(b, a) \in R\right\}$. We say that $L$ is a stage labeling of $F$ if $L_{i n}$ is conflict-free, $L_{i n} \cup L_{i n}^{+}$is maximal with respect to set inclusion and $L(a)=$ out for all $a \in A \backslash L_{i n}$.

We also make use of the notions of transitive closure of a relation and restriction of a relation to a subset of its domain.

Definition 4. Let rel be a relation. We define the transitive closure of rel to be the smallest set rel* such that $r e l \subseteq \mathrm{rel}^{*}$ and if $(a, b),(b, c) \in \mathrm{rel}^{*}$, then $(a, c) \in r e l^{*}$.

Definition 5. Let $A, B$ be sets, $A^{\prime} \subseteq A$ and $R \subseteq A \times B$. We define the restriction of $R$ to $A^{\prime}$ to be:

$$
R \downarrow_{A^{\prime}}= \begin{cases}\left\{(a, b) \in R \mid a, b \in A^{\prime}\right\} & \text { if } A=B \\ \left\{(a, b) \in R \mid a \in A^{\prime}\right\} & \text { otherwise }\end{cases}
$$

The definition of restriction handles separately two cases: if the domain and range of the relation are the same, it then applies the restriction to both of them, for example in the case of the attack relation of an AF. In the case where the domain and range are different sets, it only performs the restriction on the domain set, for example in the case of a labeling function.

In the introduction, we have pointed out that retrieving the set of complete labelings from the preferred and grounded labelings alone is not feasible. We now provide a concrete example of two argumentation frameworks with the same preferred and grounded labelings, but different complete labelings.

Example 1. Consider the two AFs $F_{1}$ and $F_{2}$ depicted in Fig. 1. Both have $\{(a$, undec $)$, $(b$, undec $),(c$, undec $),(d$, undec $)\}$ as their grounded labeling, and $\{(a$, in $),(b$, out $)$, $(c$, in $),(d$, out $)\}$ and $\{(a$, out $),(b$, in $),(c$, out $),(d$, undec $)\}$ as their preferred labelings. While these are also all the complete labelings for $F_{1}, F_{2}$ also has $\{(a$, in $),(b$, out $)$, $(c$, undec $),(d$, undec $)\}$ as a complete labeling which is neither preferred nor grounded. Hence, given nothing other than the preferred and grounded labelings of a framework, it is not feasible to always accurately retrieve the set of complete labelings. 

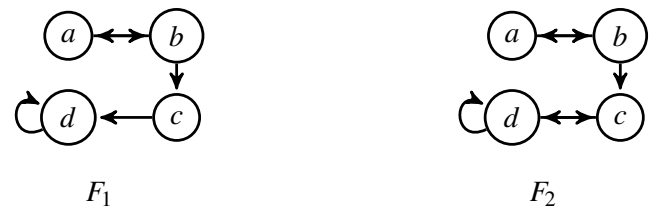

Fig. 1 Two AFs with the same preferred and grounded labelings but different complete labelings.

\section{Update relations}

Standard labeling semantics provide a direct relation between an argumentation framework and a set of labeling functions, which attach to each argument exactly one label. We will now define update relations, which formalize the idea that the final labelings can be determined in a step-wise fashion. For this purpose, we introduce epistemic labelings, which associate with each argument a nonempty set of labels from $\{$ in,out, undec $\}$. The intuitive idea is that at a certain step in the update process, the set of labels associated with an argument tells us which labels we consider possible for this argument at this step. The steps in an update relation can be interpreted as moves in a dialogue, or as steps in an algorithm, or as learning a framework, or otherwise. Our dynamic semantic framework does not depend on such particular interpretations.

Notice that it makes little sense to separate the labeling function from the underlying framework, as the labeling is meaningless without it. We will hence consider pairs of argumentation framework and labeling functions.

Definition 6. We define a labeled argumentation framework (LAF) to be a pair $(\langle A, R\rangle, L a b)$ where $\langle A, R\rangle$ is a finite argumentation framework and $L a b$ a function from $A$ to $\mathscr{P}(\mathbb{L}) \backslash\{\emptyset\}$, called an epistemic labeling. Additionally, let $\mathbb{F}$ be the class of all labeled argumentation frameworks.

Observe that a labeling function cannot assign the empty set of labels to an argument, as the set of labels represents the possible final labels for that argument, and thus the empty set would mean that no label can be attached to it, which prevents us from having a final labeling for the framework.

We now introduce the notions of initial and final labeled frameworks, which correspond to the starting point and endpoint of a labeling process. In an initial LAF, every label is possible for each argument, while in a final LAF, every argument is assigned a singleton set of labels, representing the fact that a unique label has been selected.

Definition 7. Let $F=(\langle A, R\rangle, L a b)$ be a LAF. If for all $a \in A, \operatorname{Lab}(a) \in\{\{$ in $\},\{$ out $\}$, $\{$ undec $\}$, we say that $F$ is final. If for all $a \in A, \operatorname{Lab}(a)=\mathbb{L}$, we say that $F$ is initial.

Note that there is a one-to-one correspondence between the epistemic labelings $L a b$ of the final LAFs $(\langle A, R\rangle, L a b)$ and the 3-labelings of $\langle A, R\rangle$. This one-to-one correspondence can be formally defined by constructing singletons out of a given 3-labeling as follows: 
Definition 8. Let $\langle A, R\rangle$ be an AF and $L$ a 3-labeling of $\langle A, R\rangle$, define the epistemic labeling $T(L)$ by $T(L)(a):=\{L(a)\}$ for all $a \in A$.

In this section with the basic definitions of our approach, we will be careful to make the formal distinction between a 3-labeling $L$, the corresponding epistemic labeling $T(L)$ and the corresponding final $\mathrm{LAF}(\langle A, R\rangle, T(L))$. In order to improve readability, we will not always make this distinction in later section, but instead identify the 3-labeling $L$ with the corresponding epistemic labeling $T(L)$ and the corresponding final LAF $(\langle A, R\rangle, T(L))$. For example, we might speak of an LAF being a complete labeling of a given argumentation framework, even though formally a complete labeling is a 3-labeling.

We now define a precision ordering on the LAFs based on the subset relation between the argument multi-labels, such that the final LAFs are the most precise and the initial LAFs are the least precise. Note however that only LAFs with the same underlying AF are comparable.

Definition 9. Let $F=(\langle A, R\rangle, L a b)$ and $F^{\prime}=\left(\left\langle A^{\prime}, R^{\prime}\right\rangle, L a b^{\prime}\right)$ be two labeled argumentation frameworks. We say that $F$ is at least as precise as $F^{\prime}\left(F \geq_{p} F^{\prime}\right)$, iff $\langle A, R\rangle=\left\langle A^{\prime}, R^{\prime}\right\rangle$, and for all $a \in A, \emptyset \subset \operatorname{Lab}(a) \subseteq \operatorname{Lab}^{\prime}(a)$. We say that $F$ is more precise than $F^{\prime}\left(F>_{p} F^{\prime}\right)$ iff $F \geq_{p} F^{\prime}$ and $F \neq_{p} F^{\prime}$.

We will now define the central notion of this paper, namely update relations, i.e. relations between LAFs which, starting from an initial LAF, monotonically increase precision, until a fixpoint is reached, at which point the LAF should be final and correspond to a desired output.

Definition 10. We say that $u p d \subseteq \mathbb{F} \times \mathbb{F}$ is an update relation iff:

- for all $F^{\prime} \in \mathbb{F}$ such that $u p d\left(F, F^{\prime}\right), F^{\prime} \geq_{p} F$;

- if $\operatorname{upd}(F, F)$, then $F$ is final.

Notice that by the definition of $\geq_{p}$, if $F$ is final then $\operatorname{upd}\left(F, F^{\prime}\right)$ implies $F=F^{\prime}$.

We now define correspondence between update relations and direct semantics that formalizes the idea that an update relation can be viewed as a step-wise procedure that gives rise to a certain direct semantics. For this we first need an auxiliary definition.

Definition 11. Let $R e l$ be a relation on $\mathbb{F}$ and $F$ an LAF. We say that $F$ is reachable in $R e l$ iff there exists an initial LAF $F_{i}$ such that $\left(F_{i}, F\right) \in \operatorname{Rel}^{*}$. We say that $F$ is a reachable fixpoint in $\operatorname{Rel}$ iff $F$ is reachable in $\operatorname{Rel}$ and $(F, F) \in$ Rel.

Definition 12. Let upd be an update relation and sem a semantics. We say that upd gives rise to sem iff for each 3-labeling $L$ of $\langle A, R\rangle,(\langle A, R\rangle, T(L))$ is a reachable fixpoint in upd iff $L$ is a sem labeling of $\langle A, R\rangle$.

The following theorem, which easily follows from Definition 10, provides a simple way of combining two given update relations to yield a third update relation: 
Lemma 1. If upd $d_{1}$ and $u p d_{2}$ are update relations, then $\operatorname{upd}_{1} \cup \mathrm{upd}_{2}$ is an update relation.

In Section 4 we will present an example where combining two update relations with a union operation gives us not only the union of the final labelings reachable by either of them, but also additional labelings. This means that the semantics that $u p d_{1} \cup u p d_{2}$ gives rise to is not necessarily induced by the semantics that $u p d_{1}$ and $u p d_{2}$ separately give rise to.

We are now interested in the comparison of updates in terms of precision increase per step, i.e. in the granularity of update relations. The idea is that an update relation is more granular than another if it takes more steps to reach its final LAFs. First of all, notice that such a comparison only makes sense for updates which output the same final LAF, i.e. updates which give rise to the same semantics.

Definition 13. Let upd be an update relation. We define the restriction of upd to relevant paths $(\overline{u p d})$ to be the set of pairs in upd that are in some upd-path from an initial to a final LAF.

Definition 14. Let $u p d_{1}$ and $u p d_{2}$ be two update relations. We say that $u p d_{1}$ is at least as fined-grained as $u p d_{2}\left(u p d_{1} \geq_{g} u p d_{2}\right)$ iff $\overline{u p d}_{1}^{*} \supseteq \overline{u p d}_{2}$.

We then abstractly define the most fine-grained update relation for a given labeling semantics.

Definition 15. Let sem be a labeling semantics. We define $m f g_{\text {sem }}$ to be the smallest update relation such that for all update relations upd that give rise to sem, we have $m f g_{\text {sem }} \geq_{g}$ upd.

Lemma 2. For every standard semantics, there exists a unique $\mathrm{mfg} g_{\text {sem }}$.

Proof. Define $m f g_{\text {sem }}$ as follows: $\left(F, F^{\prime}\right) \in m f g_{\text {sem }}$ iff either $F=F^{\prime}$ is a sem labeling, or the following three properties are satisfied:

- $F^{\prime}>_{p} F$;

- $\nexists F^{\prime \prime}$ such that $F^{\prime}>_{p} F^{\prime \prime}>_{p} F$;

- there exists a final $F_{f}$ which is a sem labeling such that $F_{f} \geq_{p} F^{\prime}$.

By definition, $m f g_{\text {sem }}$ includes all possible links in any relevant path from an initial to a final LAF which encompasses a sem labeling. Hence, for any update relation upd which gives rise to sem, $\overline{\mathrm{mfg}}_{\text {sem }} * \supseteq \overline{u p d}$. Also, $m f g_{\text {sem }}$ includes by definition only pairs which are on a relevant path, as the first alternative adds the endpoints of these paths and the third item of the second alternative ensures that the pairs are on a relevant path. The first and second items of the second alternative ensure also that a minimal amount of pairs are added, making $m f g_{\text {sem }}$ as small as possible. Also, note that $m f g_{\text {sem }}$ is well-defined since we only consider finite AFs, and thus $\geq_{p}$ is finite.

In subsequent sections, we will need the following notion of a sub-framework:

Definition 16. Let $F=(\langle A, R\rangle, L a b)$ be a LAF and $S \subseteq A$. We define the subframework of $F$ generated by $S$ to be $\operatorname{Sub}(F, S)=\left(\left\langle S, R \downarrow_{S}\right\rangle, L a b \downarrow_{S}\right)$. 


\section{Case analysis: An algorithmic approach for combining preferred and grounded}

In this section, we consider update relations which give rise to the preferred and grounded semantics, and which are motivated by algorithms for computing these semantics that have been described by Dauphin and Schulz [9].

The algorithmic update relation for the grounded semantics first identifies the arguments which are only being attacked by arguments which are already labeled $\{o u t\}$, labels them as $\{i n\}$ and any argument they attack as $\{o u t\}$, and then repeats this process until no arguments can be further labeled, at which point it will label all remaining arguments as $\{$ undec $\}$.

Definition 17. For any labeled argumentation framework $F=(\langle A, R\rangle, L a b)$, we define the set of unattacked arguments to be unattacked $(F)=\{a \in A \mid \operatorname{Lab}(a) \supsetneqq$ $\{$ in $\} \wedge \forall b \in A .((b, a) \in R \rightarrow \operatorname{Lab}(b)=\{$ out $\})\}$.

In an initial AF, the set of unattacked arguments will correspond to the arguments which do not have any attackers in the framework, while for final AFs, this set will be empty since it only considers arguments which are not finally labeled.

Definition 18. We define step_grnd $\subseteq \mathbb{F} \times \mathbb{F}$ to be the relation such that

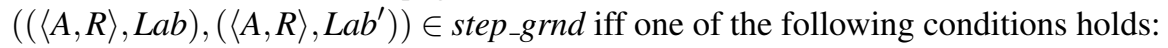

- $\operatorname{unattacked}((\langle A, R\rangle, \operatorname{Lab})) \neq \emptyset$, and $\left(\langle A, R\rangle, \operatorname{Lab}^{\prime}\right)$ is the least precise LAF that is more precise than $(\langle A, R\rangle, L a b)$ such that for all $a \in \operatorname{unattacked}((\langle A, R\rangle, L a b))$, $L a b^{\prime}(a)=\{$ in $\}$ and for all $c \in A$ such that $(a, c) \in R$ and out $\in \operatorname{Lab}(c), \operatorname{Lab}^{\prime}(c)=$ $\{$ out $\}$.

- $\operatorname{unattacked}((\langle A, R\rangle, \operatorname{Lab}))=\emptyset$, there is an $a \in A$ such that $L a b(a) \supsetneqq\{$ undec $\}$, and $\left(\langle A, R\rangle, L a b^{\prime}\right)$ is the least precise LAF that is more precise than $(\langle A, R\rangle, L a b)$ such that for all $a \in A$ such that $\operatorname{Lab}(a) \supsetneqq\{$ undec $\}, \operatorname{Lab}^{\prime}(a)=\{$ undec $\}$.

- $(\langle A, R\rangle, L a b)=\left(\langle A, R\rangle, L a b^{\prime}\right)$ is a final LAF.

Note that before labeling arguments out, we ensure that it is a possibility, e.g. by having the condition out $\in \operatorname{Lab}(c)$ in the first item of Definition 18 . While this requirement will straightforwardly be fulfilled in any reachable LAF, it is required to ensure that the increase in precision is satisfied even for those LAFs that are not reachable from an initial LAF.

The following lemma now easily follows from the above definition:

Lemma 3. step_grnd is an update relation.

The following theorem states that step_grnd does indeed have the intended property that it gives rise to the grounded labeling:

Theorem 1. step_grnd gives rise to the grounded semantics. 
Proof sketch. One can easily see that whenever step_grnd changes the label of an argument $a$ to $\{$ in $\},\{$ out $\}$ or $\{$ undec $\}$, argument $a$ is legally labeled $\{$ in $\},\{$ out $\}$ or $\{$ undec $\}$ respectively. Thus the final labeling reachable in step_grnd is a complete labeling. To show that the final labeling reachable in step_grnd is the complete labeling that maximizes undec, suppose that there is some complete labeling Lab of $\langle A, R\rangle$ and let $A^{\prime}=\{a \in A \mid \operatorname{Lab}(a)=$ undec $\}$. It is now enough to show that step_grnd never labels any $a \in A^{\prime}\{i n\}$ or $\{$ out $\}$. Consider for a proof by contradiction the first step where step_grnd does label some $a \in A^{\prime}\{$ in $\}$, respectively $\{$ out $\}$. Since $a$ is legally labeled undec in Lab, some $a^{\prime} \in A^{\prime}$ must attack $a$, so by Definitions 17 and 18, $a^{\prime}$ must already be labeled $\{$ out $\}$ in a previous step, respectively there must exist an $a^{\prime}$ which has been labeled $\{i n\}$ in a previous step, which is a contradiction.

Let us now examine step_pref, a similar update relation which computes the preferred labelings. For this, we first define the notion of minimal non-trivial admissible sets of arguments, which resembles the notion of initial-like sets [16], but takes also the partial labels into account.

Definition 19. Let $F=(\langle A, R\rangle, L a b)$ be a labeled argumentation framework. We define min_adm $(F) \subseteq \mathscr{P}(A)$ to be the set of all minimal subsets $S$ of $A$ that satisfy the following conditions:

- $S \neq \emptyset$

- for all $a \in S, \operatorname{Lab}(a) \supsetneqq\{$ in $\}$;

- for all $a, b \in S,(a, b) \notin R$;

- for all $a \in S$ and $b \in A$ such that $L a b(b) \neq\{$ out $\}$ and $(b, a) \in R$, there exists $a^{\prime} \in S$ such that $\left(a^{\prime}, b\right) \in R$.

So the function min_adm $(F)$ returns all minimal non-empty admissible sets of arguments whose label could still be changed to $\{i n\}$. The update relation step_pref proceeds with a process similar to the one in the step_grnd update, iteratively labeling $\{$ in $\}$ all arguments with all attackers $\{$ out $\}$, and then labeling all arguments attacked by those as $\{$ out $\}$. The difference lies in the case where unattacked $(F)$ is empty, where the preferred update relation looks for minimal non-trivial admissible sets, label them $\{$ in $\}$ and arguments they attack $\{$ out $\}$.

Definition 20. We define step_pref $\subseteq \mathbb{F} \times \mathbb{F}$ to be the relation such that $\left((\langle A, R\rangle, L a b),\left(\langle A, R\rangle, L a b^{\prime}\right)\right) \in$ step pref iff one of the following conditions holds:

- unattacked $((\langle A, R\rangle, L a b)) \neq \emptyset$, and $\left(\langle A, R\rangle, L a b^{\prime}\right)$ is the least precise LAF that is more precise than $(\langle A, R\rangle, L a b)$ such that for all $a \in \operatorname{unattacked}((\langle A, R\rangle, \operatorname{Lab}))$, $\operatorname{Lab}^{\prime}(a)=\{$ in $\}$ and for all $c \in A$ such that $(a, c) \in R$ and $o u t \in \operatorname{Lab}(c), \operatorname{Lab}^{\prime}(c)=$ $\{$ out $\}$.

- $\quad$ unattacked $((\langle A, R\rangle, L a b))=\emptyset$, and for some $S \in \min \_a d m(F),\left(\langle A, R\rangle, L a b^{\prime}\right)$ is the least precise LAF that is more precise than $(\langle A, R\rangle, L a b)$ such that for all $a \in S, L a b^{\prime}(a)=\{$ in $\}$ and for all $c \in A$ such that $(a, c) \in R$ and out $\in \operatorname{Lab}(c)$, $\operatorname{Lab}^{\prime}(c)=\{$ out $\}$. 
- unattacked $((\langle A, R\rangle, L a b))=$ min_adm $(F)=\emptyset$, and there is an $a \in A$ such that $L a b(a) \supsetneqq\{$ undec $\}$, and $\left(\langle A, R\rangle, L a b^{\prime}\right)$ is the least precise LAF that is more precise than $(\langle A, R\rangle, L a b)$ such that for all $a \in A$ such that $L a b(a) \supsetneqq\{$ undec $\}, \operatorname{Lab}^{\prime}(a)=$ $\{$ undec $\}$.

- $(\langle A, R\rangle, L a b)=\left(\langle A, R\rangle, L a b^{\prime}\right)$ is a final LAF.

The following lemma now easily follows from the above definition:

Lemma 4. step_pref is an update relation.

The following theorem, which can be proved in a similar way as Theorem 1, states that step_pref has the intended property that it gives rise to the preferred labeling:

Theorem 2. step_pref gives rise to the preferred semantics.

We now find the interesting result that combining these two update relations with a union operation gives us not only the union of the final labelings reachable by either of them, but also the complete labelings which are neither grounded nor preferred:

Theorem 3. step_grnd $\cup$ step_pref gives rise to the complete semantics.

Proof sketch. One can easily see that any final labeling reachable in step_grnd $\cup$ step_pref is a complete labeling, as the two update relations preserve the legality of argument labels. So we only prove that each complete labeling $L a b$ is reachable in step_grnd $\cup$ step_pref.

Let $F=(\langle A, R\rangle, L a b)$ be an initial $\mathrm{LAF}$ and $L a b_{c}$ the complete labeling we want to reach. First, apply either step_grnd or step_pref until we reach $F^{\prime}$ where the set of unattacked arguments is empty. At this point, the set $S$ of in arguments is the grounded extension, and thus these arguments must also be in in $L a b_{c}$, since the grounded extension is the intersection of all complete extensions (this follows from it being the unique smallest complete extension). Let $S^{\prime}$ be the set of arguments which are in in $L a b_{c}$ but not $\{i n\}$ in $F^{\prime} . S \cup S^{\prime}$ forms an admissible set, since it is a complete extension. Hence, there is a minimal, non-empty subset of $S^{\prime}, S_{1}^{\prime}$, such that $S \cup S_{1}^{\prime}$ is admissible. There is an edge in the relation step_pref which labels the arguments in $S_{1}^{\prime}$ as in and any argument they attack as out, according to Def. 20 second item. The rest of the arguments in $S^{\prime}$ are labeled in via Def. 20, either with the first item, or again with the second item as above. Once we have reached the LAF where all in arguments from $L a b_{c}$ are $\{i n\}$ and any argument they attack $\{$ out $\}$, we can make a step with step_grnd following Def. 18, second item, to label all remaining arguments as $\{$ undec $\}$. We have then reached the fixpoint $F_{f}=\left(\langle A, R\rangle, T\left(L a b_{c}\right)\right)$, as desired.

Example 2. Let us examine the initial LAF $F=(\langle A, R\rangle, L a b)$ where $A=\{a, b, c, d\}$, $R=\{(a, b),(b, a),(b, c),(c, d),(d, c)\}$. Since unattacked $(F)=\emptyset$, step_grnd will send $F$ to the fixpoint where all arguments are labeled $\{$ undec $\}$. This is depicted 


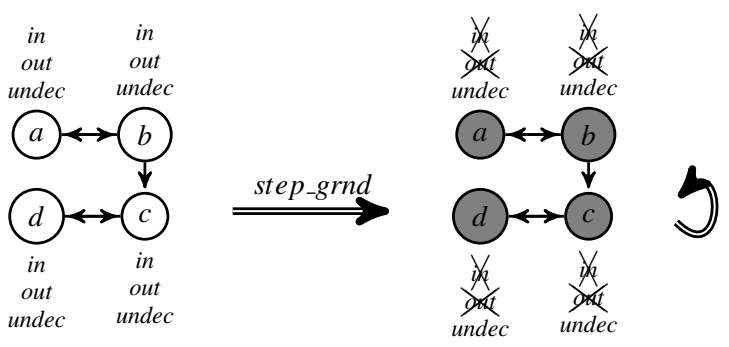

Fig. 2 Example path from the initial LAF $F$ to the corresponding final LAF in step_grnd.

in Fig. 2.

Let us now consider the same LAF $F$ under the step_pref update relation this time. Again, unattacked $(F)=\emptyset$, but min_adm $(F)=\{\{a\},\{b\},\{d\}\}$. The relation hence branches out in three paths. Let us focus the path with $\{a\}$. So the relation step_pref sends $F$ to the LAF $F_{\text {pref } 1}$ where $a$ is $\{i n\}$ and $\mathrm{b}$ is $\{$ out $\}$, as depicted in Fig. 3. unattacked $\left(F_{\text {pref } 1}\right)=\emptyset$, but min_adm $\left(F_{\text {pref } 1}\right)=\{\{c\},\{d\}\}$, which gives us once again two possible directions in which to branch out. We will examine the one which selects $\{c\}$. This then gives us the final fixpoint $F_{\text {pref } 2}=\left(\langle A, R\rangle, L a b_{\text {pref } 2}\right)$, where $\operatorname{Lab}_{\text {pref2 } 2}(a)=\operatorname{Lab}_{\text {pref } 2}(c)=\{$ in $\}$ and $\operatorname{Lab}_{\text {pref } 1}(b)=\operatorname{Lab} b_{\text {pref1 }}(d)=\{$ out $\}$.

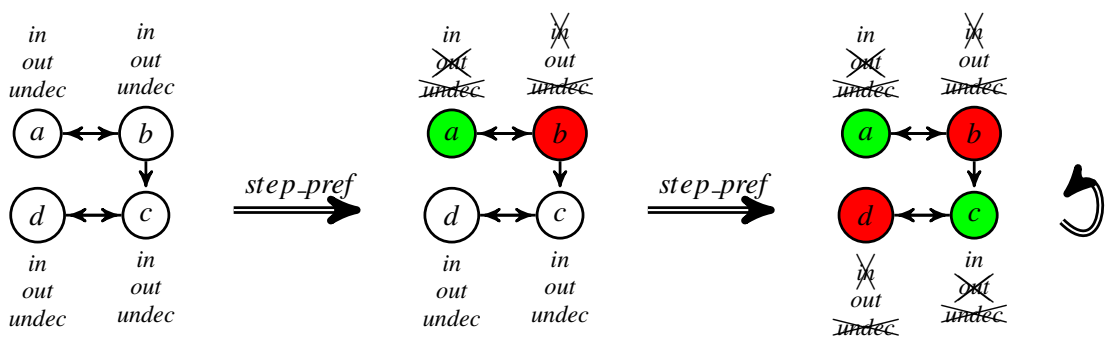

Fig. 3 Example path from the initial LAF $F$ to one of the corresponding final LAFs in step_pref.

We now consider the union of both relations. We can first send $F$ to $F_{p r e f 1}$ using the same step from step_pref as above. However this time we can apply step_grnd to $F_{\text {pref } 1}$, and since unattacked $\left(F_{\text {pref } 1}\right)=\emptyset$, the remaining arguments $c$ and $d$ are assigned the $\{$ undec $\}$ label, sending $F_{\text {pref } 1}$ to the fixpoint $F_{\text {comp }}$, where $a$ is $\{$ in $\}, b$ is $\{$ out $\}$ and $c, d$ are $\{u n d e c\}$. Notice that $F_{\text {comp }}$ corresponds to a complete labeling of $F$ which is neither preferred nor grounded. This situation is depicted in Fig. 4. 


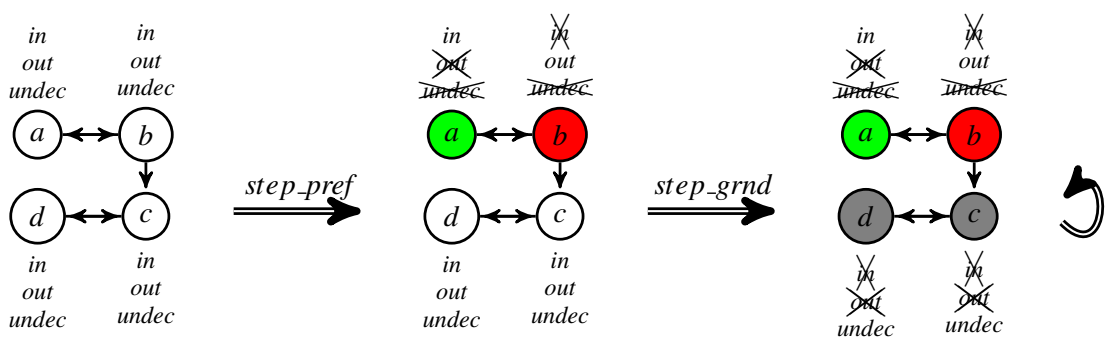

Fig. 4 Example path from the initial LAF $F$ to one of the corresponding final LAFs in step_grnd $\cup$ step_pref which neither update can reach by itself.

\section{Merging semantics through the most fined-grained update relation}

In the previous section, we have shown that we can obtain the complete semantics by taking the union of two algorithmically motivated update relations giving rise to the grounded and the preferred semantics respectively. The success of this approach was dependent on the details of the algorithmic update relations that we defined, so it cannot be generalized to combine arbitrary semantics. In this section, we want to generalize our methodology to make it applicable to the combination of arbitrary semantics. For this purpose, we will examine a way to combine any two standard semantics via their most fine-grained update and a combination operation we call merging.

\subsection{Merging preferred and grounded}

If we were to attempt to combine $m f g_{\text {pref }}$ and $m f g_{g r n d}$ by simply taking their union, as we have done in the algorithmic approach, it follows from their definition that we would simply obtain as reachable fixpoints the labelings which are either preferred or grounded. The main issue is that $m f g_{\text {pref }}$ and $m f g_{\text {grnd }}$ are not applicable to LAFs which do not agree with some final LAF of that semantics.

For an example of this issue, we consider again the same LAF $F$ as in Example 2. Suppose we want to reach the same complete labeling that we reached in Figure 4, i.e. the one in which $a$ is $\{i n\}, b$ is $\{$ out $\}$, and $c$ and $d$ are $\{$ undec $\}$. We could start by doing those six steps of $m f g_{\text {grnd }}$ that are compatible with the complete labeling that we want to reach, as depicted in Figure 5, yielding the intermediate LAF $F^{\prime}$. Now we would like to apply $m f g_{\text {pref }}$ to $F^{\prime}$ in order to delete the undec-labels from $a$ and $b$. However, $m f g_{\text {pref }}$ cannot be applied at all to $F^{\prime}$, as $F^{\prime}$ is not compatible with any preferred labeling of $F$.

So instead of just taking the union of $m f g_{\text {pref }}$ and $m f g_{g r n d}$, we will define a more complicated operation called the merge of two update relations, which we denote by 


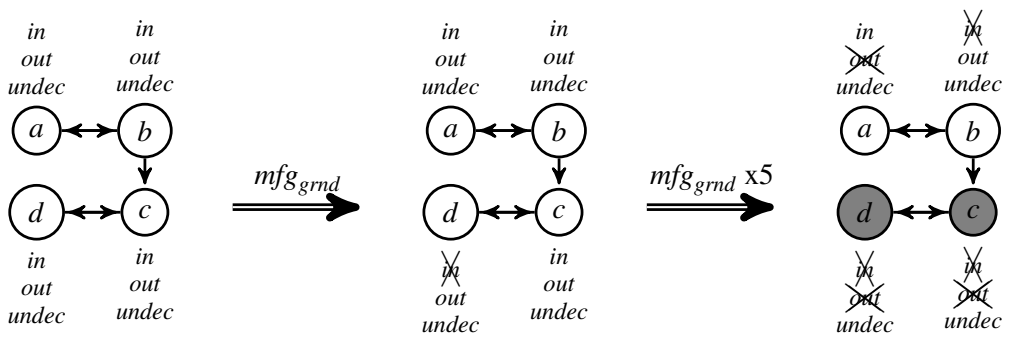

Fig. 5 Example path from the initial LAF $F$ to an intermediate LAF $F^{\prime}$ in $m f g_{\text {grnd }}$.

$u p d_{1} \uplus u p d_{2}$. The idea is that once neither $m f g_{\text {pref }}$ nor $m f g_{\text {grnd }}$ allow us to get closer to a desired complete labeling, we will focus on a particular sub-framework and draw analogies with another framework which also contains that sub-framework. This operation resembles the way input is imposed in multi-sorted argumentation semantics [2]. The details of this approach are somewhat complicated, so let us first sketch the approach by seeing how it can be applied to the example that we just looked at.

The idea is that we focus on the set $S=\{a, b\}$ of arguments, as we want to remove labels from $a$ and $b$. In order to work with $m f g_{\text {pref }}$ on the sub-framework $\operatorname{Sub}\left(F^{\prime}, S\right)$ induced by $S$, we consider an alternative framework $F_{2}$ that also has $\operatorname{Sub}\left(F^{\prime}, S\right)$ as a sub-framework, but to which $m f g_{\text {pref }}$ can be applied. A suitable choice of $F_{2}$ is depicted on the left in Figure 6. Now we apply $m f g_{\text {pref }}$ twice to $F_{2}$ as depicted in Figure 6, removing the labels from $a$ and $b$ that we wanted to remove. If certain conditions are satisfied, we may import the changes we have made to $F_{2}$ back to $F$, as depicted in Figure 7.

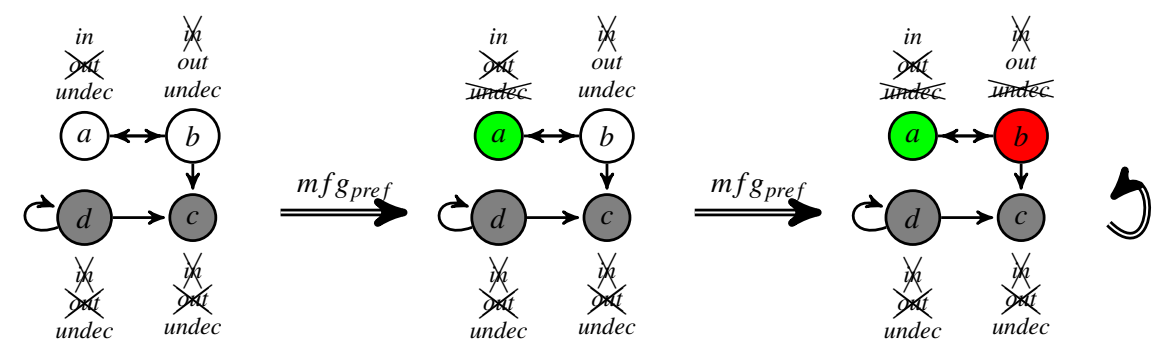

Fig. 6 Example path on a parallel $F_{2}$ framework with $S=\{a, b\}$ and $I=\{c\}$, where $m f g_{\text {pref }}$ is applicable.

Now what are the conditions that need to be satisfied in order to allow for this import of changes from one framework to another? In order to describe these conditions, we need to split the original framework into three parts, based on sets of arguments: 


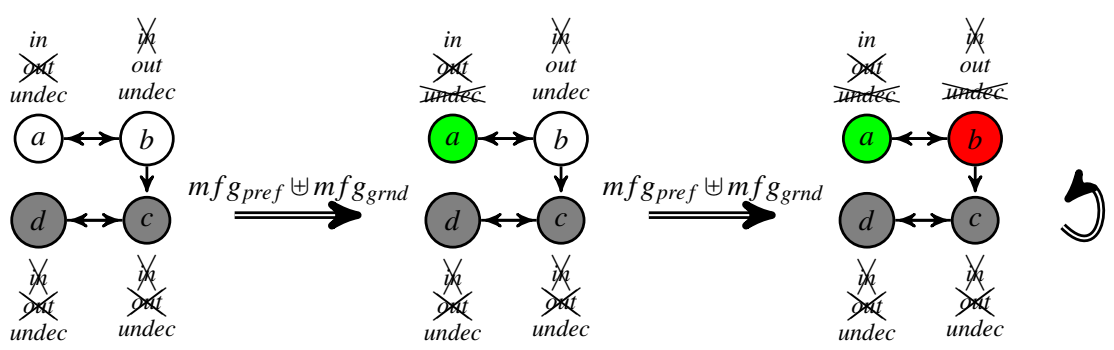

Fig. 7 Importing the steps made in Fig. 6 into $F^{\prime}$ allows us to reach a complete labeling which is neither grounded nor preferred.

- $S$, the arguments we will focus on;

- I, called the interface, which is a set of arguments which already have a maximally precise label (i.e. a singleton) and which separate the set $S$ from the rest of the framework;

- $A \backslash(S \cup I)$, the rest of the framework, on which the two frameworks may differ.

The basic idea is that in order to import some change that an update relation $m f g_{\text {sem }}$ can make on $F_{2}$ to the LAF $F$, we have to choose $F_{2}$ in such a way that in both $F$ and $F_{2}$, the interface $I$ separates $S$ from the rest of the framework. Furthermore, we have to choose $F_{2}$ in such a way that $m f g_{\text {sem }}$ can actually be applied to $F_{2}$, which is only possible if the maximally precise labels of the arguments in $I$ are possible labels for these arguments in $F_{2}$ under the semantics sem.

We are now ready to present the formal definition of the merge $u p d_{1} \uplus u p d_{2}$ :

Definition 21. Let $u p d_{1}$ and $u p d_{2}$ be two update relations. We define the merge of $u p d_{1}$ and $u p d_{2}\left(u p d_{1} \uplus u p d_{2}\right)$ as the smallest relation such that:

1. upd $\uplus \uplus$ upd $_{2} \supseteq$ upd $_{1} \cup$ upd $_{2}$;

2. For $F=(\langle A, R\rangle, L a b)$ and $F^{\prime}=\left(\langle A, R\rangle, L a b^{\prime}\right),\left(F, F^{\prime}\right) \in u p d_{1} \uplus u p d_{2}$ if there exist disjoint sets $S, I \subseteq A$ and two LAFs $F_{2}=\left(\left\langle A_{2}, R_{2}\right\rangle, L a b_{2}\right)$ and $F_{2}^{\prime}=$ $\left(\left\langle A_{2}, R_{2}\right\rangle, L a b_{2}^{\prime}\right)$ such that the following conditions are satisfied:

a. $\left(F_{2}, F_{2}^{\prime}\right) \in u_{u p d} \cup u_{1} d_{2}$;

b. $\operatorname{Sub}\left(F_{2}, S \cup I\right)=\operatorname{Sub}(F, S \cup I)$;

c. $\forall s \in S, \forall a \in A \backslash(I \cup S),(s, a),(a, s) \notin R, R_{2}$;

d. $\forall a \in I, \operatorname{Lab}(a)=\operatorname{Lab}_{2}(a)$ is a singleton;

e. $L a b_{2}^{\prime} \downarrow_{S} \neq L a b_{2} \downarrow_{S}$;

f. $L a b_{2}^{\prime} \downarrow_{A_{2} \backslash S}=L a b_{2} \downarrow_{A_{2} \backslash S}$;

g. $\operatorname{Sub}(F, A \backslash S)$ is reachable by $u p d_{1} \uplus u p d_{2}$;

h. $L a b^{\prime} \downarrow_{S}=L a b_{2}^{\prime} \downarrow_{s}$.

3. if $F$ is final and reachable by $u p d_{1} \uplus u p d_{2}$, then $(F, F) \in u p d_{1} \uplus u p d_{2}$.

Given the complexity of this definition, let us explain it a bit more: Item 1 expresses the fact that we can still perform any step which is available in either one of 
the base updates. However, as we have seen previously, this is not enough in order to obtain meaningful combinations of most fine-grained updates, which is why we have item 2. Given a labeled argumentation framework $F$, additional changes are potentially possible if we can identify two disjoint sets of arguments $S$ and $I$, where $S$ is the set of arguments we are interested in and where the update will be occurring and $I$ is a fully-labeled interface between $S$ and the rest of the framework, meaning that no argument in $S$ attacks nor is attacked by an argument in $A \backslash(S \cup I)$. Once such sets have been identified, we observe other labeled argumentation frameworks $F_{2}$ which also contain $S \cup I$ with the same structure and epistemic labels but can differ in structure and labels in the rest of the framework. If an update with $u p d_{1} \cup u p d_{2}$ is possible in such a framework, we then allow this change to be imported into $F$ to produce $F^{\prime}$. In more details, sub-item $a$ specifies that there must be a $u p d_{1} \cup u p d_{2}$ step which relates $F_{2}$ to $F_{2}^{\prime}$. Sub-item $b$ ensures that the parallel framework $F_{2}$ agrees with $F$ on the structure and epistemic labels of $S \cup I$. Sub-item $c$ guarantees that there are no connections between $S$ and $A \backslash(S \cup I)$ in neither $F$ nor $F_{2}$. Sub-item $d$ ensures that $I$ is fully labeled, which is required in order to ensure the well-behavior of the merge operation. The idea is that once this interface has been fully labeled by one of the two updates, if we can modify $A \backslash(S \cup I)$ in order to make sense of these labels for the second update, then we can also perform steps from this second update inside $S$, and then by perhaps modifying $A \backslash(S \cup I)$ again we can switch back to using the first update again and so on. Sub-item $e$ ensures that change happens inside $S$, while sub-item $f$ ensures that no change is made outside of $S$, so that change happens in $S$ and exclusively there. Sub-item $g$ provides an additional restriction on the partitioning to ensure that for an argument $i$ in the interface $I$ which has a justification $a \in S$ for its label which hasn't been assigned yet, we do not introduce a new justification in $A \backslash(S \cup I)$ for $i$ 's label and hence allow for a different label to be assigned to $a$, leaving $i$ with no justification for its label in $F^{\prime}$. This is clarified in Example 3. Sub-item $h$ simply specifies that the change made in the parallel LAF be imported into the original one to produce $F^{\prime}$, and combined with the sub-item $e$ entails that a change within $S$ is necessary between $F_{2}$ and $F_{2}^{\prime}$. Finally, with item 3 we ensure that reachable final frameworks are also fixpoints, which is needed since the second item of the definition does not produce any fixpoints, as it requires some change to happen in the LAF with the first sub-item.

Example 3. Consider the AF $F$ depicted in Fig. 8. Since $\{(a$, in $),(b$, out $),(c$, out $)$, $(d$, in $),(e, o u t)\}$ is a preferred labeling, it is possible to assign the out label to $c$ via $m f g_{\text {pref }}$, as well as the in label to $a$ and the out label to $b$. From there, it would be possible to set $I=\{c\}$ and $S=\{d, e\}$, allowing one to import changes from the parallel framework $F^{\prime}$ depicted in Fig. 9. Here, a few steps in $m f g_{\text {grnd }}$ would assign the undec label to $d$ and $e$. This would however produce a labeling where $c$ is out, but has no reason to be labelled so, since $d$ is undec. This kind of scenario is prevented by item g) of Def. 21 as no sub-LAF consisting of $\{a, b, c\}$ where $c$ is out is reachable with $m f g_{\text {pref }}$ or $m f g_{\text {grnd }}$. Thus, item g) forces what we informally refer to as a justification for the interface's label to be either part of it, or contained in $S$. 


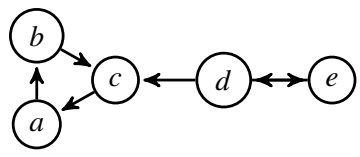

Fig. 8 Example AF $F$ to illustrate the need for item g) in Def. 21

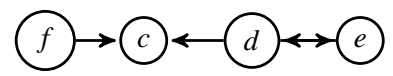

Fig. 9 AF $F^{\prime}$ parallel to $F$ from Fig. 8. Here $c$ is out due to $f$, allowing $m f g_{\text {grnd }}$ to assign the undec label to both $d$ and $e$.

In definition 21 , we have defined the merge between two arbitrary update relations. In this paper, we always apply this merge operation to two maximally finegrained update relations and focus on the semantics that the resulting update relation gives rise to. In this way, the notion of a merge between two update relations gives rise to the following notion of a merge between two argumentation semantics:

Definition 22. Given two argumentation semantics $\mathrm{sem}_{1}$ and $\mathrm{sem}_{2}$, we define $\mathrm{sem}_{1} \uplus$ $s e m_{2}$ to be the semantics that $m f g_{s e m_{1}} \uplus m f g_{\text {sem }_{2}}$ gives rise to.

We originally motivated the definition of the merge operation with the goal to combine the grounded and preferred semantics to yield the complete semantics. The following theorem establishes that this is indeed the case for the merge operation as we have defined it.

Theorem 4. preferred $\uplus$ grounded $=$ complete.

Proof. By Definition 22, we need to show that $m f g_{\text {pref }} \uplus m f g_{\text {grnd }}$ gives rise to the complete semantics. So we need to prove that every complete labeling is a reachable fixpoint in $m f g_{\text {pref }} \uplus m f g_{\text {grnd }}$ and that every labeling that is a reachable fixpoint in $m f g_{\text {pref }} \uplus m f g_{\text {grnd }}$ is a complete labeling. We start by proving that every complete labeling is a reachable fixpoint in $m f g_{\text {pref }} \uplus m f g_{\text {grnd }}$ :

Let $A F=\langle A, R\rangle$ be an argumentation framework, and let $L$ be the complete labeling of $A F$ we want to reach. We want to show that $F_{f}=\left(\langle A, R\rangle, L a b_{f}\right)$ is a reachable fixpoint, where $L a b_{f}=T(L)$. Let $C=\{a \in A \mid L(a)=i n\}, I=\{b \in$ $A \mid L(a)=$ out $\}$ and $S=A \backslash(C \cup I)$. Consider the LAF $F_{i}=\left(\langle A, R\rangle, L a b_{i}\right)$, where for all $a \in C, L a b_{i}(a):=\{i n\}$, for all $b \in I, L a b_{i}(b):=\{o u t\}$, and for all $c \in S$, $L a b_{i}(c):=\{$ in, out, undec $\}$. Since $L$ is a complete labeling, $C$ is admissible, so there exists a preferred labeling where all arguments in $C$ are in. Thus $F_{i}$ is reachable with $m f g_{\text {pref }}$.

We now want to apply item 2 of Definition 21 to $F_{i}$ multiple times in order to remove all the in and out labels from the arguments in $S$. For this purpose, we choose a "new" argument $z$, i.e. an argument $z \notin A$, and consider the LAF $F_{2}=\left(\left\langle A_{2}, R_{2}\right\rangle, L a b_{2}\right)$ where $A_{2}=(A \backslash C) \cup\{z\}, R_{2}=R \downarrow_{A_{2}} \cup\{(z, a) \mid a \in I\}$ and $L a b_{2}=L a b \downarrow_{A_{2}} \cup\{(z,\{i n\})\}$. Consider the final LAF $F_{2 f}=\left(\left\langle A_{2}, R_{2}\right\rangle, L a b_{2 f}\right)$, where for all $a \in A_{2} \backslash S, L a b_{2 f}(a)=L a b_{2}(a)$ and for all $a \in S, L a b_{2 f}(a)=\{$ undec $\}$. 
We want to show that $F_{2 f}$ is grounded. For this purpose, we first establish that $F_{2 f}$ is complete, i.e. that all labels in $F_{2 f}$ are legal labels: $z$ is unattacked and is therefore legally labeled in in $F_{2 f}$. All arguments in $I$ are attacked by $z$, so they are legally labeled out in $F_{2 f}$. Furthermore, since $C$ does not defend any arguments it does not contain, every argument in $S$ is attacked by at least one other argument in $S$. Additionally, the only in argument, $z$, does not attack any arguments in $S$. Thus the arguments in $S$ are legally labeled undec in $F_{2 f}$. Therefore, $F_{2 f}$ is a complete LAF, and since the only in argument, $z$, has to be labeled in, it is also grounded.

Therefore $F_{2 f}$ is reachable in $m f g_{\text {grnd }}$ from $F_{2}$. So by multiple applications of $m f g_{\text {pref }} \uplus m f g_{\text {grnd }}$, using item 2 of Def 21, one can reach $F_{f}$ from $F_{i}$. Since $F_{f}$ is final, $F_{f}$ is a fixpoint, and thus $F_{f}$ is a reachable fixpoint.

So far, we have shown that every complete labeling is a reachable fixpoint in $m f g_{\text {pref }} \uplus m f g_{\text {grnd }}$. Now we still need to show that every labeling that is a reachable fixpoint in $m f g_{\text {pref }} \uplus m f g_{\text {grnd }}$ is a complete labeling:

Let $F=(\langle A, R\rangle, L a b)$ be a reachable LAF in $m f g_{\text {pref }} \uplus m f g_{\text {grnd }}$. We show by induction on $|A|$ that there exists a final complete LAF which is at least as precise as $F$.

Induction hypothesis 1: Assume that for every LAF $F^{\prime}=\left(\left\langle A^{\prime}, R^{\prime}\right\rangle, L a b^{\prime}\right)$ such that $\left|A^{\prime}\right|<|A|$ and $F^{\prime}$ is reachable in $m f g_{\text {pref }} \uplus m f g_{\text {grnd }}$, there exists a final complete LAF which is at least as precise as $F^{\prime}$.

We now use a second induction on the steps required to reach $F$.

Base case: $F$ is initial. Since there always exists a complete labeling for any framework, there exists a final complete LAF more precise than $F$.

Inductive step: $F$ is not initial, but is reached in $m f g_{\text {pref }} \uplus m f g_{\text {grnd }}$ through an LAF $F^{*} \neq F$ for which the required property holds. In other words, we have the following induction hypothesis for $F^{*}$ :

Induction hypothesis 2: Assume that for $F^{*}=\left(\langle A, R\rangle, L a b^{*}\right)$ such that $F^{*} \neq F$ and $\left(F^{*}, F\right) \in m f g_{\text {pref }} \uplus m f g_{\text {grnd }}$, there exists a final complete $\operatorname{LAF} F_{f}^{*}=\left(\langle A, R\rangle, L a b_{f}^{*}\right)$ such that $F^{*} \leq_{p} F_{f}^{*}$.

We distinguish three cases:

1. $\left(F^{*}, F\right) \in m f g_{\text {pref }}$. Then, by the definition of $m f g_{\text {pref }}$, there exists a final LAF which represents a preferred labeling of $\langle A, R\rangle$ and is at least as precise as $F$. Since preferred labelings are also complete, we are done.

2. $\left(F^{*}, F\right) \in m f g_{\text {grnd }}$. Similarly to the case above, it follows from the definition of $m f g_{\text {grnd }}$ that there exists a complete final LAF which is at least as precise as $F$.

3. $\left(F^{*}, F\right) \notin m f g_{\text {pref }} \cup m f g_{\text {grnd }}$. Since $F^{*} \neq F$, the item 2 of Definition 21 must be satisfied. In other words, there exist disjoint sets $S, I \subseteq A$ and two LAFs $F_{2}$ and $F_{2}^{\prime}$ that satisfy the conditions $a$ to $h$ from item 2 of Definition 21. By condition $a,\left(F_{2}, F_{2}^{\prime}\right) \in m f g_{\text {pref }} \cup m f g_{\text {grnd }}$, so by the same reasoning as in cases 1 and 2 above, we can conclude that there exists a final LAF $F_{f 2}=\left(\left\langle A_{2}, R_{2}\right\rangle, L a b_{f 2}\right)$ which is complete and at least as precise as $F_{2}^{\prime}$. Also, by condition $g, F_{s}=$ $\operatorname{Sub}\left(F^{*}, A \backslash S\right)$ is reachable by $m f g_{\text {pref }} \uplus m f g_{\text {grnd }}$, and by condition $e, S \neq \emptyset$, i.e. 
$|A \backslash S|<|A|$. So by induction hypothesis 1 , there exists a final complete LAF $F_{s f}=\left(\left\langle A \backslash S, R \downarrow_{A \backslash S}\right\rangle, L a b_{s f}\right)$ such that $F_{s f} \geq_{p} F_{s}$.

We now construct the final LAF $F_{f}=\left(\langle A, R\rangle, L a b_{f}\right)$ as follows: For all $a \in$ $A \backslash S, \operatorname{Lab}_{f}(a):=\operatorname{Lab}_{s f}(a)$, and for all $a \in S, \operatorname{Lab}_{f}(a)=\operatorname{Lab}_{f 2}(a)$. From the construction of $F_{f}$ and conditions $f$ and $h$ of Definition 21, it follows that $F_{f}$ is more precise than $F$. To complete the proof, we now still need to show that $F_{f}$ is a complete labeling, i.e. that all arguments in $A$ are legally labeled in $F_{f}$.

According to the definition of legal labeling (Definition 2), a labeling being legal depends only on the label of the arguments it is directly attacking or attacked by. According to condition $c$ of Definition 21, the only arguments which are attacking or attacked by arguments in $S$ are in $S \cup I$. The arguments in $S$ are legally labeled in $F_{2 f}$, and thus they are also legally labeled in $F_{f}$, since $\operatorname{Sub}\left(F_{2 f}, S \cup I\right)=\operatorname{Sub}\left(F_{f}, S \cup I\right)$. Similarly, since the arguments in $A \backslash(S \cup I)$ are legally labeled in $F_{s f}$, they are also legally labeled in $F_{f}$. Now take an arbitrary $a \in I$. We distinguish three cases:

a. $\operatorname{Lab}_{f}(a)=\{$ out $\}$ : Then, since $F_{s f}$ is complete, there exists an argument $b \in A \backslash S$ such that $(b, a) \in R$ and $\operatorname{Lab}_{f}(b)=\{$ in $\}$. So $a$ is legally out in $F_{f}$.

b. $L a b_{f}(a)=\{$ in $\}$ : Then, since $F_{s f}$ is complete, for all $b \in A \backslash S$ such that $(b, a) \in R, L a b_{f}(b)=\{$ out $\}$. Also, since $F_{2 f}$ is complete, for all $b \in S$ such that $(b, a) \in R, L a b_{f}(b)=\{$ out $\}$. Hence, $a$ is legally in in $F_{f}$.

c. $L a b_{f}(a)=\{$ undec $\}$ : Then, since since $F_{s f}$ is complete, for all $b \in A \backslash S$ such that $(b, a) \in R, \operatorname{Lab}_{f}(b) \neq\{i n\}$, and for at least one such $b, L a b_{f}(b)=$ $\{$ undec $\}$. Also, since $F_{2 f}$ is complete, for all $b \in S$ such that $(b, a) \in R$, $L a b_{f}(b) \neq\{$ in $\}$. Hence, $a$ is legally undec in $F_{f}$.

So all arguments in $F_{f}$ are legally labeled and thus $F_{f}$ is complete. Hence, there exists a final complete LAF which is at least as precise as $F$.

Therefore, for all reachable LAFs, there exists a complete LAF which is at least as precise. Since $m f g_{\text {pref }} \uplus m f g_{\text {grnd }}$ is an update relation, every reachable fixpoint is final, and thus every reachable fixpoint is complete.

\subsection{Defining new semantics via merging}

The merge operation defined in Definition 22 can be used to combine two arbitrary argumentation semantics to yield another argumentation semantics. So far, we have shown that merging grounded and preferred semantics yields the complete semantics. In this section, we show how applying this merge operation to other pairs of semantics gives rise to completely new argumentation semantics.

First, notice that the second part of the proof of Theorem 4 only makes use of the fact that the labelings reached at the different stages are complete, but not of any other properties particular to preferred or grounded. Hence, the merge of any two complete-based semantics is a complete-based semantics itself, i.e. all the labelings it returns are also complete. 
Theorem 5. Let sem se $_{1}$ and $\mathrm{sem}_{2}$ be two complete-based argumentation semantics. Then sem $_{1} \uplus \mathrm{sem}_{2}$ is also a complete-based semantics.

For example, by merging stable and grounded, we obtain labelings which are complete. However, in this case, we do not recover all complete labelings as we did when merging grounded and preferred. Let us examine this short example to see why.

Example 4. Consider the following AF, which we call $F$ :

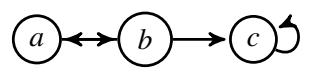

Using $m f g_{\text {stab }} \uplus m f g_{\text {grnd }}$, one can reach the labelings $\{(a$, out $),(b, i n),(c$, out $)\}$ and $\{(a$, undec $),(b$, undec $),(c$, undec $)\}$. However, suppose we wish to reach the complete labeling $L a b=\{(a, i n),(b, o u t),(c$, undec $)\}$. Since there is no stable labeling, we cannot make any steps via $m f g_{\text {stab }}$ from the initial LAF. Also, attempting to find a similar framework from which one could import changes, will not work at this point where the LAF is initial, because the interface $I$ would have to be empty, which only works for disconnected AFs.

Hence, one can only make steps in $m f g_{\text {grnd }}$ in order to reduce $c$ 's epistemic labeling to $\{$ undec $\}, A$ 's to $\{$ in, undec $\}$ and $B$ 's to $\{$ out undec $\}$. This, however, is as close as one can get to $L a b$ using $m f g_{\text {stab }} \uplus m f g_{\text {grnd }}$. Any $F_{2}$ satisfying the conditions of item 2 of Definition 21 must have $I=\{c\}$. In this case, the set $S$ on which we want to make changes would have to be $\{a, b\}$. But then $I \cup S$ includes all arguments, so that $F_{2}$ would have to be identical to $F$, so that we cannot use item 2 of Definition 21 to make any change that we cannot already make with item 1 of Definition 21.

Therefore, $L a b$ is unreachable with $m f g_{\text {stab }} \uplus m f g_{\text {grnd }}$.

An interesting note to make is that all labelings reachable by $m f g_{\text {stab }} \uplus m f g_{\text {grnd }}$ are complete, according to Theorem 5 , and hence this combination provides a novel complete-based semantics which returns more labelings than both the stable semantics and the grounded semantics.

Similarly, the merge of the semi-stable and grounded semantics returns a novel complete-based semantics. One can check this by replacing stable by semi-stable in the situation described in Example 4: The desired complete labeling is still unreachable.

As motivated in the introduction, we are also interested in the following research question related to combining features of naive-based and complete-based semantics: Is there a sensible semantics that allows one to locally make choices for some unattacked odd or even cycles while not making choices for other unattacked odd or even cycles. Given our methodology for merging semantics, an obvious candidate for such a semantics is stage $\uplus$ grounded, i.e. the semantics resulting from merging the stage semantics with the grounded semantics. By considering its application to an example, we show that this semantics does indeed have this feature. 


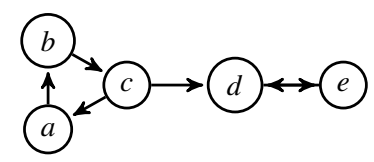

Example 5. Consider the following AF, which we call $F^{\prime}$ :

The stage labelings of $F^{\prime}$ are

$$
\begin{aligned}
& L a b_{1}=\{(a, \text { in }),(b, \text { out }),(c, \text { out }),(d, \text { in }),(e, \text { out })\}, \\
& L a b_{2}=\{(a, \text { in }),(b, \text { out }),(c, \text { out }),(d, \text { out }),(e, \text { in })\}, \\
& L a b_{3}=\{(a, \text { out }),(b, \text { in }),(c, \text { out }),(d, \text { in }),(e, \text { out })\}, \\
& L a b_{4}=\{(a, \text { out }),(b, \text { in }),(c, \text { out }),(d, \text { out }),(e, \text { in })\}, \\
& L a b_{5}=\{(a, \text { out }),(b, \text { out }),(c, \text { in }),(d, \text { out }),(e, \text { in })\} .
\end{aligned}
$$

Its grounded labeling is $L a b_{6}=\{(a$, undec $),(b$, undec $),(c$, undec $),(d$, undec $),(e$, undec $)\}$. Additionally to these six labelings, it has three further stage $\uplus$ grounded-labelings:

$$
\begin{aligned}
& L a b_{7}=\{(a, \text { in }),(b, \text { out }),(c, \text { out }),(d, \text { undec }),(e, \text { undec })\}, \\
& L a b_{8}=\{(a, \text { out }),(b, \text { in }),(c, \text { out }),(d, \text { undec }),(e, \text { undec })\}, \\
& L a b_{9}=\{(a, \text { undec }),(b, \text { undec }),(c, \text { undec }),(d, \text { out }),(e, \text { in })\} .
\end{aligned}
$$

$L a b_{7}$ can be reached using $m f g_{\text {stage }} \uplus m f g_{\text {grnd }}$ by first applying $m f g_{\text {stage }}$ several times to reduce the epistemic labels on $a, b$ and $c$ to $\{$ in $\},\{o u t\}$ and $\{o u t\}$ respectively and then applying item 2 of Definition 21 with the interface $I:=\{c\}$, the set $S:=\{d, e\}$ and the following parallel framework $F_{2}^{7}$ :

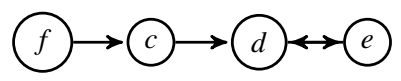

We can then apply $m f g_{\text {grnd }}$ multiple times to this parallel framework to reduce the epistemic labels of $d$ and $e$ to $\{u n d e c\}$ and import these changes to the labeling on the main framework $F^{\prime}$ using item 2 of Definition 21. $L a b_{8}$ can be reached using $m f g_{\text {stage }} \uplus m f g_{\text {grnd }}$ in a similar way using the same parallel framework.

$L a b_{9}$ can be reached using $m f g_{\text {stage }} \uplus m f g_{\text {grnd }}$ by first applying $m f g_{\text {stage }}$ several times to reduce the epistemic labels on $d$ and $e$ to $\{$ out $\}$ and $\{i n\}$ respectively and then applying item 2 of Definition 21 with the interface $I:=\{d\}$, the set $S:=\{a, b, c\}$ and the following parallel framework $F_{2}^{9}$ :

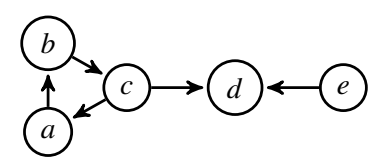


We can then apply $m f g_{\text {grnd }}$ multiple times to this parallel framework to reduce the epistemic labels of $a, b$ and $c$ to $\{u n d e c\}$ and import these changes to the labeling on the main frameowrk $F^{\prime}$ using item 2 of Definition 21.

The stage semantics forces us to make a choice on the odd cycle $\{a, b, c\}$, and unless we choose to accept the argument $c$ that attacks the even cycle, we are also forced to make a choice on the even cycle $\{d, e\}$. In the grounded semantics, there are no choices and all arguments become undecided. In stage $\uplus$ grounded, we can combine these features of stage and grounded: We can for example choose $a$ from the odd cycle, but stay undecided about the arguments in the even cycle - this possible choice is formalized by $\mathrm{Lab}_{7}$.

So stage $\uplus$ grounded allows one to locally make choices for some unattacked odd or even cycles while not making choices for other unattacked odd or even cycles. It thus provides a positive answer to our third research question from the introduction.

\section{Conclusion and future work}

In this paper we introduce a dynamic approach to combine two argumentation semantics to yield a third one. In particular, we provide a formal environment for the analysis of step-wise relations between labeled framework with an increase in the label precision, whose reachable fixpoints correspond to some standard direct semantics. We define and discuss two approaches to combining two given update relations to yield a third update relation, an approach based on algorithmically motivated update relations and an approach based on merging maximally finegrained update relations. For both approaches, we examine how to obtain update relations for the complete labeling by combining update relations for the preferred and grounded labelings. Furthermore, we have defined novel semantics using the merge approach, including a semantics that meaningfully combines features of naive-based and complete-based semantics.

Our paper gives rise to various topics for further research. Concerning the combination of argumentation semantics, many questions remain. Further new semantics can be defined using our approach, and properties of the newly defined semantics can be studied systematically using the principle-based approach $[4,14]$.

Though we introduced our update relations to combine argumentation semantics, we believe that this dynamic semantics framework can be used for other applications as well. Most importantly, one of the main challenges in formal argumentation is the gap between graph based semantics and dialogue theory. Our more dynamic semantics framework may be used to decrease or even close the gap. In particular, in dialogue each statement may increase the knowledge and thus the set of arguments of participants. This is also related to the formalization of learning in the context of formal argumentation. Moreover, an important approach in argumentation semantics is the SCC recursive scheme. This scheme can be represented naturally

using update relations. Various algorithms have been proposed for argumentation 
semantics, and these algorithmic approaches may be expressed naturally using update relations. Work has also been done on dynamic modifications to be made on a framework in order to enforce a certain set of arguments to become an extension, or prevent it from being so [6,7]. Parallels could be made between their work and the combination operation presented in this paper. Finally, the principle based analysis of argumentation semantics can be extended to the more fine grained update relations.

\section{References}

1. Ryuta Arisaka, Ken Satoh, and Leendert van der Torre. Anything you say may be used against you in a court of law. In Artificial Intelligence and the Complexity of Legal Systems (AICOL). Springer, 2018.

2. Pietro Baroni, Guido Boella, Federico Cerutti, Massimiliano Giacomin, Leendert van der Torre, and Serena Villata. On the Input/Output behavior of argumentation frameworks. Artificial Intelligence, 217:144-197, 2014.

3. Pietro Baroni, Martin Caminada, and Massimiliano Giacomin. An introduction to argumentation semantics. The Knowledge Engineering Review, 26(4):365-410, 2011.

4. Pietro Baroni and Massimiliano Giacomin. On principle-based evaluation of extension-based argumentation semantics. Artificial Intelligence, 171(10-15):675-700, 2007.

5. Pietro Baroni, Massimiliano Giacomin, and Giovanni Guida. Scc-recursiveness: a general schema for argumentation semantics. Artificial Intelligence, 168(1-2):162-210, 2005.

6. Ringo Baumann and Gerhard Brewka. Expanding argumentation frameworks: Enforcing and monotonicity results. COMMA, 10:75-86, 2010.

7. Sylvie Coste-Marquis, Sébastien Konieczny, Jean-Guy Mailly, and Pierre Marquis. On the revision of argumentation systems: Minimal change of arguments statuses. $K R, 14: 52-61$, 2014.

8. Marcos Cramer and Mathieu Guillaume. Two approaches to dialectical argumentation: Admissible sets and argumentation stages. In Proceedings of the International Conference on Computational Models of Argument (COMMA), 2018.

9. Jeremie Dauphin and Claudia Schulz. Arg Teach - A Learning Tool for Argumentation Theory. In Tools with Artificial Intelligence (ICTAI), 2014 IEEE 26th International Conference on, pages 776-783. IEEE, 2014.

10. Phan Minh Dung. On the acceptability of arguments and its fundamental role in nonmonotonic reasoning, logic programming and n-person games. Artificial intelligence, 77(2):321-357, 1995.

11. Sarah Alice Gaggl and Wolfgang Dvořák. Stage semantics and the SCC-recursive schema for argumentation semantics. Journal of Logic and Computation, 26(4):1149-1202, August 2016.

12. Massimiliano Giacomin. Handling Heterogeneous Disagreements Through Abstract Argumentation. In International Conference on Principles and Practice of Multi-Agent Systems, pages 3-11. Springer, 2017.

13. Tjitze Rienstra, Alan Perotti, Serena Villata, Dov M Gabbay, and Leendert van der Torre. Multi-sorted argumentation. In International Workshop on Theorie and Applications of Formal Argumentation, pages 215-231. Springer, 2011.

14. Leendert van der Torre and Srdjan Vesic. The principle-based approach to abstract argumentation semantics. In Pietro Baroni, Dov Gabbay, Massimiliano Giacomin, and Leendert van der Torre, editors, Handbook of Formal Argumentation. College Publications, 2018.

15. Bart Verheij. Two approaches to dialectical argumentation: Admissible sets and argumentation stages. In Proceedings of the biannual International Conference on Formal and Applied Practical Reasoning (FAPR) workshop, pages 357-368. Universiteit, 1996. 
16. Yuming Xu and Claudette Cayrol. Initial sets in abstract argumentation frameworks. Journal of Applied Non-Classical Logics, pages 1-20, 2018. 